\title{
Tecnologia no cuidado como instrumento de diagnóstico do câncer no colo do útero: um relato de experiência
}

\author{
Diagnosing instrument for cervical cancer: an experience report
}

La tecnología en el cuidado como herramienta para diagnosticar el cáncer de cuello uterino: un informe de experiencia

Jessica de Souza Pereira ${ }^{1 *}$, Érika Patrícia Oliveira de Oliveira ${ }^{1}$, Jessica de Lira Teixeira ${ }^{1}$, Luciana Emanuelle de Aviz ${ }^{1}$, Karina Borges da Silva ${ }^{1}$, lanny Ferreira Raiol ${ }^{1}$, Shirley Aviz de Miranda ${ }^{1}$, Maria Rute de Souza Araujo', Juliana Raiyanni Sousa Neto'.

\section{RESUMO}

Objetivo: Relatar o desenvolvimento de uma atividade educativa com uso de uma tecnologia no cuidado sobre diagnóstico e tratamento no exame Papanicolau em uma Estratégia Saúde da Família (ESF). Relato de Experiência: $O$ primeiro momento foi o levantamento bibliográfico para maior aprofundamento e conhecimentos científicos sobre o tema. Posteriormente foi elaborada uma tecnologia em forma de disco com informações sobre diagnóstico e tratamento do câncer de colo de útero. Por fim, foi desenvolvida uma ação educativa em uma ESF no município de Belém-PA sobre a prevenção do câncer de colo de útero e apresentação da tecnologia com informações das lesões mais leve à mais grave e as condutas que o enfermeiro deve realizar diante da lesão. Participaram nove profissionais de saúde, os quais relatam os desafios e dificuldades que os profissionais enfrentam no atendimento das mulheres e suas experiências e desafios encontrados no rastreamento, identificação e condutas em casos alterados de exame de Papanicolau. Considerações finais: Ressalta-se que a atividade educativa proporcionou importantes construções de conhecimentos, favorecendo o fortalecimento do processo ensino-aprendizagem, além de estreitar as relações entre ensino e comunidade, além de que a disponibilidade da tecnologia para o serviço possibilita ampliação do cuidado à população assistida.

Palavras-chave: Neoplasias do colo do útero, Diagnóstico, Estratégia saúde da família.

\begin{abstract}
Objective: To report the development of an educational activity using a technology in the care of diagnosis and treatment in the Pap smear in a Family Health Strategy (FHS). Experience Report: The first moment was the bibliographic survey to deepen scientific knowledge on the subject. Subsequently a disc-shaped technology was developed with information on diagnosis and treatment of cervical cancer. Finally, an educational action was developed in an FHS in the city of Belém-PA on the prevention of cervical cancer and presentation of the technology with information on the lightest to most serious injuries and conduct that nurses should perform in the face of the injury. Nine health professionals participated, who report the challenges and difficulties professionals face in the care of women and their experiences and challenges found in the screening, identification and management of altered Pap smears. Final considerations: It is noteworthy that the educational activity provided important constructions of knowledge, favoring the strengthening of the teaching-learning process, in addition to strengthening the relationship between teaching and the community, and the availability of technology for the service enables the expansion of care to assisted population.
\end{abstract}

Key words: Cervical neoplasms, Diagnosis, Family health strategy.

\footnotetext{
${ }^{1}$ Centro Universitário Metropolitano da Amazônia (UNIFAMAZ), Belém-Pará. *E-mail: je8362@gmail.com
} 


\section{RESUMEN}

Objetivo: Informar el desarrollo de una actividad educativa utilizando una tecnología en el cuidado del diagnóstico y tratamiento en la prueba de Papanicolaou en una Estrategia de Salud Familiar (FHS). Informe de experiencia: El primer momento fue la encuesta bibliográfica para profundizar el conocimiento científico sobre el tema. Posteriormente, se desarrolló una tecnología de disco con información sobre el diagnóstico y el tratamiento del cáncer de cuello uterino. Finalmente, se desarrolló una acción educativa en un FHS en la ciudad de Belém-PA sobre la prevención del cáncer de cuello uterino y la presentación de la tecnología con información sobre las lesiones más leves y más graves y la conducta que las enfermeras deben realizar frente a la lesión. Nueve profesionales de la salud participaron, informando sobre los desafíos y dificultades que enfrentan los profesionales en el cuidado de las mujeres y sus experiencias y desafíos encontrados en la detección, identificación y manejo de las pruebas de Papanicolaou alteradas. Consideraciones finales: Cabe destacar que la actividad educativa proporcionó importantes construcciones de conocimiento, favoreciendo el fortalecimiento del proceso de enseñanza-aprendizaje, además de fortalecer la relación entre la enseñanza y la comunidad, y la disponibilidad de tecnología para el servicio permite la expansión de la atención a población asistida.

Palabras clave: Neoplasias cervicales, Diagnóstico, Estrategia de salud familiar.

\section{INTRODUÇÃO}

O Câncer de Colo de Útero (CCU) é considerado um problema de saúde pública no país. É a terceira causa de morte entre as mulheres no mundo. Segundo o Instituto Nacional do câncer (INCA, 2017) estimase que o risco é de 15,85 em cada 100 mil mulheres, sendo uma das maiores taxas de prevalência e mortalidade da doença. Do total de óbitos por essa afecção em todo o mundo, cerca de $85 \%$ deles sucederam nos países menos desenvolvidos devido aos agravos que propiciam maior incidência da doença (COSTA FKM, et al. 2017).

Os principais fatores de risco para o desenvolvimento do CCU é o início precoce da atividade sexual, múltiplos parceiros sexuais, baixa condição socioeconômicos, hábitos de vida inapropriado, tabagismo, higiene íntima inadequada, desconhecimentos da progressão da doença e a infecção causada pelo Papiloma Vírus Humano (HPV), sendo que os principais subtipos são os 16 e 18, responsáveis pelo CCU, no qual a contaminação ocorre através de relações sexuais desprotegidas. O HPV está presente em quase todos os casos de câncer uterino. Aproximadamente $20 \%$ da população do mundo estão infectados pelo HPV e a maioria dessa população apresenta infecções assintomáticas e transitórias (SOUZA AF e COSTA LHR, 2015).

A alteração de Neoplasia Intraepitelial Cervical (NIC) 1 ou displasia leve tem uma taxa de retrocesso maior que $50 \%$, enquanto para NIC 3 tem um avanço de $11 \%$ e $1 \%$ respectivamente, o que a torna um avanço muito baixo. Lesões do HPV-induzidas de baixo grau são muito comuns, estão sujeitas a muitas modificações para serem consideradas pré-câncer: mulheres com HPV/NIC 1 possui um risco mínimo de evoluir para câncer invasor, não necessitando de propedêutica e tratamento invasivo. Já as mulheres com NIC 2 ou 3 devem ser tratadas corretamente, com a exérese da lesão da forma mais apropriada, pelo alto risco de evoluir para uma neoplasia invasora (SANTOS UM, SOUZA SEBD, 2014).

De acordo com o Ministério da Saúde (BRASIL, 2017), o exame Papanicolau deve ser realizado em mulheres que já iniciaram a atividade sexual, principalmente aquelas na faixa etária de 25 a 59 anos. A Estratégia Saúde da Família (ESF) tem uma grande importância na ampliação da oferta do exame em todo o território nacional, além de disponibilizar vacinas contra HPV gratuitamente pelo Sistema Único e Saúde (SUS), como método de prevenção (ANDRADE et al., 2014).

Diversas estratégias vêm sendo adotadas para garantir o acesso das mulheres ao programa de rastreamento do câncer do colo do útero. A ESF, por ser um modelo de assistência constituído por equipes responsáveis por fazer buscas ativas e o mapeamento do território, favorece o acesso da população ao programa (MISTURA C, et al., 2011). 
Neste sentido, a ESF permite uma aproximação da Equipe de Saúde com a mulher, por atuar na integridade, nas ações educativas e visitas domiciliares. Essa busca possibilita a identificação ativa das mulheres elegíveis para o rastreamento e tratamento, portanto o profissional tem um papel importante nas unidades como orientar e estimular com que essas mulheres adotem o exame Papanicolau em uma unidade básica de saúde como uma rotina anual (GIRIANELLI VR, et al., 2014)

Destaca-se que a ausência de estratégias educacionais, acaba dificultando as práticas preventivas e uns dos principais fatores é a falta de conhecimento em relação da doença e da importância do exame Papanicolau, os aspectos culturais, medo da dor, vergonha, falta de informação/sensibilização dos profissionais de saúde, atitudes irrelevantes do parceiro e pudores relacionados a exposição do corpo (COSTA FKM, et al., 2017).

A equipe de enfermagem tem um papel importante, pois, é essencial que o enfermeiro seja crítico-reflexivo, assim, possibilitando um olhar holístico, capaz de desenvolver uma assistência de qualidade e resolutividade de forma humanizada e integral para que possa intervir diante os possíveis problemas da população, a enfermagem deve realizar educação em saúde por meio de ações de prevenção, promoção e recuperação a saúde. Desta forma, conscientizar a população e atingindo uma maior cobertura no rastreamento do CCU (VIANA MRP, et al., 2013).

A capacitação do profissional, humanização e o diálogo da equipe é de total importância, pois, passa às mulheres confiança, com isso às mesmas se sentem acolhidas retornando periodicamente a unidade para 0 atendimento integral. Entretanto, para atingir a cobertura de qualidade no serviço prestado por sua equipe, 0 enfermeiro deve executar outras atribuições como promoção e educação a saúde continuada, assim contribuindo para que o serviço na unidade seja prestado integralmente e com qualidade (AMARAL AF, et al., 2014).

As ferramentas tecnológicas tais como, as que apresentam metodologias inovadoras que possibilitam auxiliar e/ou intervir nas práticas educativas na comunidade, além do mais, estimula-os para a buscar por respostas de problemas complexos. A tecnologia não deve ser entendida apenas como produto e/ou procedimento, mas também como algo que possa facilitar e/ou contribuir para as práticas educativas e compartilhamento de conhecimentos. (SILVEIRA RSO, et al., 2016).

Há vários tipos de tecnologias do cuidado em saúde que são utilizados na prática dos profissionais em saúde, as tecnologias e o conhecimento são processos que estão em constante organização e reorganização pelo ser humano para melhor adequação e transformação da realidade que o cerca. Essas podem trazer importantes transformações à pesquisa, ensino, assistência e no processo de trabalho da enfermagem. Tendo um papel importante para o profissional, pois, elas podem facilitar os processos gerenciais e ações práticas do cuidado em enfermagem. A partir das dificuldades observadas, percebeu-se que algumas tecnologias poderiam facilitar e qualificar o processo de trabalho nas unidades de saúde (CAMPOS NBD, 2014).

O objetivo geral desse trabalho é relatar o desenvolvimento de uma atividade educativa sobre o uso de uma tecnologia no cuidado sobre diagnóstico e tratamento do Câncer de colo do útero entre profissionais de uma Estratégia Saúde da Família.

\section{RELATO DE EXPERIÊNCIA}

Trata-se de um relato de experiência sobre a elaboração de uma tecnologia no cuidado de diagnóstico e tratamento do CCU para ser utilizada entre profissionais que atuam na Atenção Básica em Saúde e sobre a realização de uma atividade educativa com a temática entre profissionais que atuam em uma Estratégia Saúde da Família (ESF).

Num segundo momento, foi elaborada a ferramenta tecnológica, com o intuito de auxiliar e facilitar o atendimento dos profissionais na ESF. A tecnologia é lúdica e didática em forma de disco que contém informações sobre diagnóstico e tratamento do câncer de colo de útero, de acordo com a nova classificação citológica estabelecida pelo Instituto Nacional de Câncer (BRASIL, 2016). O objetivo no desenvolvimento 
desta ferramenta foi de auxiliar o profissional de saúde no diagnóstico e na conduta inicial frente aos resultados exames de citologia oncótica.

O disco possui três etapas resumidas das recomendações iniciais que o profissional de saúde deve seguir mediante as alterações citológicas de acordo com as diretrizes brasileiras para o rastreamento do câncer do colo do útero (BRASIL, 2016). A primeira trata-se dos diagnósticos citopatologicos que são: Células escamosas atípicas de significado indeterminado (ASCUS), Células glandulares atípicas de significado indeterminado (AGC), Células atípicas de origem indefinida (AOI), Lesão de Baixo Grau (LSIL), Lesão de Alto Grau (HSIL), Lesão intraepitelial de alto grau não podendo excluir microinvasão, Carcinoma escamoso invasor e Adenocarcinoma in situ (AIS) ou invasor. A segunda etapa constitui-se na orientação aos profissionais na conduta inicial diante do resultado do exame citopatologico e de acordo com a faixa etária da cliente. A terceira etapa conduz ao tratamento que a paciente deverá realizar diante do estadiamento da doença.

Figura 1 - Tecnologia rastreamento CCU.

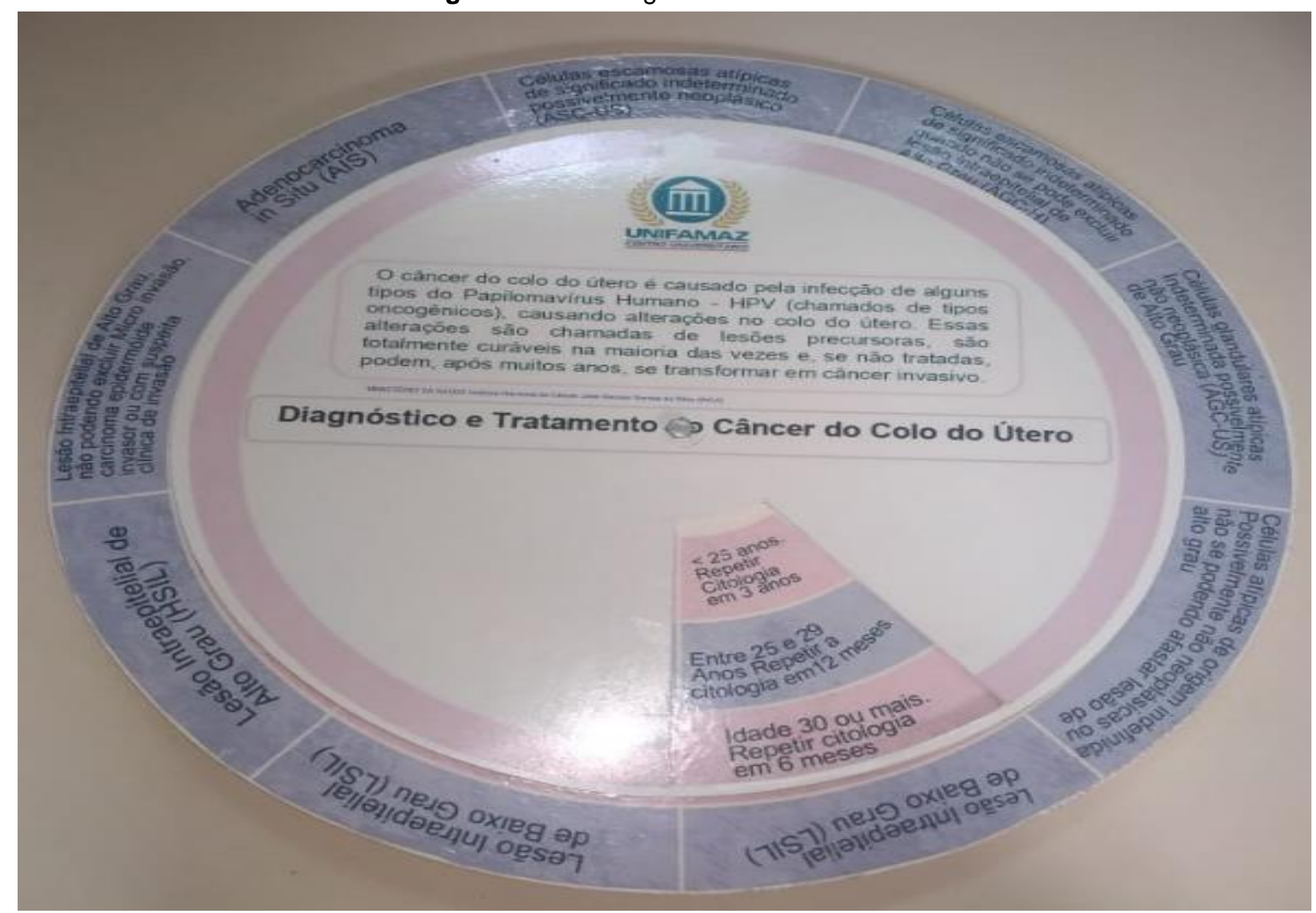

Fonte: Pereira JS, et al., 2019

O terceiro momento foi a apresentação da tecnologia por meio de uma ação educativa na ESF, localizada no estado do Pará. O público alvo foi a equipe de saúde da ESF que estavam presente. A equipe da Unidades de saúde é formada por dois enfermeiros, dois médicos, dois técnicos de enfermagem, e seis Agentes Comunitários de Saúde (ACS). A ESF atende um total de 2.700 pessoas cadastradas.

Inicialmente foi realizada uma breve introdução do que é o CCU, os fatores que desencadeiam essa patologia e suas formas de prevenção, logo após apresentada foi demonstrado como utilizar a tecnologia em forma de disco. Na qual, contém informações das lesões mais leves até lesões mais graves e quais as condutas, que o enfermeiro deve realizar diante da lesão que a paciente apresentar, com isso agilizando e facilitando o entendimento para os profissionais e para as usuárias do serviço. 
Participaram da ação educativa 9 (nove) profissionais, dentre eles 3 (três) homens e 5 (cinco) mulheres, um médico, dois enfermeiros, dois técnicos de enfermagem e quatro ACS. Após a apresentação da tecnologia discutiu-se com eles, os desafios e as dificuldades que os profissionais enfrentam no atendimento das mulheres, os profissionais relataram sobre suas experiências e desafios encontrados.

Houve uma boa participação e interação da equipe de saúde, com apresentação da tecnologia os mesmos mostraram-se muito satisfeitos com a ferramenta, enalteceram que ela seria de grande utilidade na unidade de saúde, pois facilitaria no momento das consultas, orientações e nas educações em saúde.

\section{DISCUSSÃO}

Compreende-se que o desenvolvimento da atividade educativa e disponibilização do disco para a equipe da ESF possibilitou trocas importantes de vivencias e conhecimentos. Todos mostraram-se interessados na temática e referiram a importância da ferramenta tecnologia no auxílio do cuidado e no rastreamento do CCU na Atenção básica (AMARAL AF, et al., 2014).

De acordo com Jakobczynsk J et al (2018) para que rastreamento do CCU ocorra com eficácia é essencial que análise das amostras do exame citopatológico, seja realizada sem erros e com qualidade, assim evitando resultados falso-negativos. Uma amostra coletada corretamente apresenta células devidamente distribuídas, fixadas e coradas, mas para isso é necessário que o profissional coletor tenha capacidade e conhecimento de uma coleta correta. Com isso, é necessário que os profissionais de saúde que atuam na prevenção do câncer de colo de útero tenham capacitação e qualificação continua para a realizar a coleta das amostras do material cervicovaginal. Essa capacitação faz toda a diferença na qualidade e confiança dos resultados.

Durante a atividade, foi verbalizado diversas barreiras e dificuldades para o rastreamento do CCU, com destaque para o pouco conhecimento das mulheres sobre os fatores de risco do HPV. Segundo Souza AF, Costa LHR (2015) mais da metade dos casos diagnosticados de CCU estão ligados diretamente ao vírus do HPV. O vírus é transmitido principalmente por relação sexual, contato direto com a pele ou mucosa infectada. Apesar da amplitude do problema em questão, infelizmente ainda pouco se fala sobre o assunto entre as mulheres, mesmo tendo hoje ferramentas de rastreamento e de tratamento eficazes os índices de mulheres infectadas continuam exorbitantes, a falta de informação entre elas é uma das principais dificuldades que os profissionais encontram no combate desta doença. Além disso, o número de mulheres que realizam o exame e não retornam para buscar o resultado é muito grande, o que dificulta a cobertura de $100 \%$ do rastreamento do CCU, outros fatores que geralmente estão relacionados com a falta de procura pelo exame são: medo de um resultado positivo, vergonha de se expor, falta de sintomas aparentes, entre outros.

Um dos profissionais participantes relatou que uma das dificuldades que a equipe enfrenta na consulta de enfermagem com as usuárias é a vergonha em expor suas partes intimas e o medo que as mulheres têm em realizar os exames, pois, não sentem confiança no profissional. Segundo Leite MF, et al. (2014) algumas usuárias sofrem barreiras socioeconômica, as quais: baixa escolaridade, falta de informações, a dificuldade de acesso para a realização do exame ou quando realizam e não retornam para busca o resultado.

Em seu estudo Abreu MNS, et al. (2018) ressalta que a falta de conhecimento das mulheres sobre o vírus HPV e de sua relação com o CCU, às impedem de procurar métodos preventivos ou de detectar lesões que podem indicar a infecção com o vírus. Conforme os estudos de Amaral AF, et al. (2014) apontam que os fatores da vulnerabilidade feminina por uma infecção sexualmente transmissível (IST), a baixa escolaridade, questões socioeconômicas e também a insegurança na realização do exame aumentam quando se trata de um profissional do sexo masculino, o que dificulta ainda mais a realização/adesão do exame.

Segundo Nascimento RG e Araújo A. (2014) evidenciam que a baixa adesão na realização do exame Papanicolau é o sentimento de vergonha, questões culturais, medo do diagnostico, falta de compreensão das orientações e desconhecimento dos profissionais, falta de informações sobre o exame citopatológico do colo do útero e a espera pelos sintomas, destaca-se também que devido à sobrecarga do dia-a-dia, como o trabalho, cuidado com os filhos, falta de tempo, desleixo, falta de interesse faz com que as mulheres se tornem vítimas, prejudicando-as em suas demandas de saúde. 
Observou-se que Santos LM, Silva, AKBL (2019), relatam que apesar das estratégias nacionais para aumentar a cobertura do exame, é necessário realizar ações educativas através de palestras, rodas de conversa e orientações individuais se apresentaram com a finalidade de sensibilizar acerca da importância da realização do exame Papanicolau desde o início da vida sexual, além de estimular o comparecimento das usuárias.

Nas falas dos participantes, foi relatado por alguns ACS dúvidas em relação aos fatores que podem desencadear um câncer de colo de útero e sobre o HPV. Outro ponto que chamou a atenção foi a fala feitas pelos ACS durante a visita domiciliar de que muitas mulheres relataram não ter conhecimento sobre o exame e que não sabiam o que era o exame Papanicolau. De acordo com Ramos AL, et al. (2014) uma das atribuições do ACS é realizar busca ativa para rastreamento de mulheres e detecção precoce do CCU. No entanto, é responsabilidade do enfermeiro e médico da ESF realizar o seguimento e continuidade da linha de cuidado das mulheres com exame alterado, garantindo tratamento e acompanhamento adequado, resolutivo e contínuo. Diante das falas dos ACS foram retiradas suas dúvidas explicando a eles fatores que desencadeiam CCU, explanando também, como são realizados os exames e suas formas de prevenção e tratamento, consequentemente tenham um melhor entendimento e possam repassar informações corretas a essas mulheres.

\section{CONSIDERAÇÕES FINAIS}

Conclui-se que o desenvolvimento da atividade educativa possibilitou novas experiências e proporcionou a construção do conhecimento e estreitamento entre o ensino e comunidade. A ação educativa possibilitou ainda para todos os envolvidos na atividade mais conhecimento e novas experiências sobre o câncer no colo do útero e os fatores que influenciam na alta prevalência do CCU e nas dificuldades de rastreamento. Observou-se que a equipe se mostrou muito satisfeita com a ferramenta tecnológica, e relataram que à mesma era muito eficaz e que facilitaria na conduta do rastreamento do CCU. Os mesmos, enfatizaram sobre seus desafios e dificuldades enfrentados para fazer a cobertura de $100 \%$ do exame citopatológico, com a falta de conhecimento das mulheres em relação a patologia e não a busca pelo diagnóstico e tratamento. Ressaltase a importância da promoção da linha de cuidado das mulheres com exame alterado, garantindo tratamento e acompanhamento adequado, resolutivo e contínuo.

\section{REFERÊNCIAS}

1. ABREU MNS, et al. Conhecimento e percepção sobre o HPV na população com mais de 18 anos da cidade de Ipatinga, MG, Brasil. Revista Ciência \& Saúde Coletiva, 2018; 23(3); 849-860.

2. AMARAL AF, et al. Impacto da capacitação dos profissionais de saúde sobre o rastreamento do câncer do colo do útero em unidades básicas de saúde. Rev Bras Ginecol Obstet, 2014; 36(4);182-7

3. BATISTA RCL. Papel da enfermagem na prevenção, diagnóstico e tratamento do câncer de colo uterino: uma revisão integrativa. TCC (Trabalho de Conclusão de Curso) - Universidade de Brasília- Faculdade de Ceilândia, Distrito Federal, 2015; 22p.

4. BRASIL. Instituto Nacional de Câncer José Alencar Gomes da Silva. Coordenação de Prevenção e Vigilância. Divisão de Detecção Precoce e Apoio à Organização de Rede. 2. ed. rev. atual. - Rio de Janeiro: INCA, 2016.

5. CAMPOS NBD. As ferramentas tecnológicas auxiliando os enfermeiros no controle e prevenção do câncer do colo do útero no ambulatório de uma maternidade pública de belo horizonte. Monografia (Linhas de Cuidado em Enfermagem) - Universidade Federal de Santa Catarina, FLORIANÓPOLIS, 2014; 36 p.

6. COSTA FKM, et al. Os desafios do Enfermeiro perante a prevenção do câncer de colo do útero. Revista de gestão e saúde, 2017; 17(01); 55-62.

7. GIRIANELLI VR, et al. Os grandes contrastes na mortalidade por câncer do colo uterino e de mama no Brasil. Rev Saúde Pública 2014; 48(3):459-467

8. INSTITUTO NACIONAL DE CÂNCER- (INCA). Estimativa 2018. Incidência do Câncer no Brasil. Rio de Janeiro: INCA, 2017. Disponível em: http://www.inca.gov.br/estimativa/2018/estimativa-2018.pdf. Acesso em: 28 de outubro de 2019.

9. JAKOBCZYNSK J, et al. Capacitação dos profissionais de saúde e seu impacto no rastreamento de lesões precursoras do câncer de colo uterino, RBAC. 2018; 50(1): 80-5 
10. LEITE MF, et al. Conhecimentos e prática das mulheres sobre câncer de colo do útero de uma unidade básica de saúde. Jornal of human growth and development, 2014; 24(2): 208-213.

11. MISTURA C, et al. Papel do enfermeiro na prevenção do câncer de colo uterino na estratégia saúde da família. Revista Contexto \& Saúde, 2011; 11 (20): 1161-1164.

12. NASCIMENTO RG, ARAUJO A. Falta de periodicidade na realização do exame citopatológico do colo uterino: motivações das mulheres. Revista reme. 2014; 18(3): 557-564.

13. RAMOS AL, et al. A atuação do enfermeiro da estratégia saúde da família na prevenção do câncer de colo de útero. Revista sanare, 2014; 13 (1): 84-91.

14. SOUZA AF, COSTA LHR. Conhecimento de mulheres sobre HPV e câncer do colo do útero após consulta de enfermagem. Revista Brasileira de cancerologia, 2015; 61(4): 343-350.

15. SILVEIRA RSO, et al. Construção de Tecnologia Educativa para Incentivar Puérperas ao Aleitamento Materno. Ceará, 2016; $1: 1-3$

16. SANTOS LM, SILVA, AKBL. Câncer de colo do útero: papel do enfermeiro na prevenção e detecção precoce dessa neoplasia na atenção básica. 2019; 16 (3): 463-475.

17. SANTOS UM, SOUZA SEBD. Papanicolau: diagnóstico precoce ou prevenção do câncer cervical, uterino. Revista Baiana de Saúde Pública. 2014; 37 (4): 941.

18. VIANA MRP, et al. Formação do enfermeiro para prevenção do câncer de colo uterino. Revista enferm. UERJ, Rio de Janeiro, 2013; 21 (1): 624-630. 